CAHIERS DE

NARRATOLOGIE

\section{Cahiers de Narratologie}

Analyse et théorie narratives

$14 \mid 2008$

Prose d'idées : formes et savoirs

\title{
Écriture de soi et prose d'idées : l'exemple des Mémoires de Jean-François Marmontel
}

\section{Anne Coudreuse}

\section{(2) OpenEdition}

1 Journals

Édition électronique

URL : https://journals.openedition.org/narratologie/625

DOI : 10.4000/narratologie.625

ISSN : 1765-307X

Éditeur

LIRCES

\section{Référence électronique}

Anne Coudreuse, «Écriture de soi et prose d'idées : l'exemple des Mémoires de Jean-François Marmontel », Cahiers de Narratologie [En ligne], 14 | 2008, mis en ligne le 29 février 2008, consulté le 21 septembre 2021. URL : http://journals.openedition.org/narratologie/625; DOI : https://doi.org/ 10.4000/narratologie.625

Ce document a été généré automatiquement le 21 septembre 2021.

\section{c) (†) $९$}

Cahiers de Narratologie - Analyse et théorie narratives est mis à disposition selon les termes de la licence Creative Commons Attribution - Pas d'Utilisation Commerciale - Pas de Modification 4.0 International. 


\title{
Écriture de soi et prose d'idées : l'exemple des Mémoires de Jean- François Marmontel
}

\author{
Anne Coudreuse
}

1 Il peut sembler incongru et peu pertinent de poser la question de la prose d'idées dans ses rapports avec l'écriture de soi, et plus particulièrement avec le genre des Mémoires. Lieu de l'épanchement, du récit de soi et des faits, ce genre est-il bien adapté à la réflexion et à l'élaboration intellectuelle? Dans le chapitre qu'il consacre à la prose d'idées au XVIII siècle dans l'Histoire de la France littéraire parue aux PUF en 2006, Stéphane Pujol distingue les enquêtes philosophiques (l'essai, les formes fragmentaires, les discours et les esquisses) des genres dialogiques (la lettre d'idées, le dialogue philosophique) ${ }^{1}$. Il se garde bien de confondre dialogue et dialogisme : «Doctrinal ou réellement heuristique, «ouvert » ou fermé », le dialogue n'est pas systématiquement un modèle de littérature dialogique, si du moins l'on entend par cette expression l'exposition antidogmatique et plurielle d'une vérité ». Le terme dialogisme "engage une réflexion sur la division et la répartition des voix, sur la polyphonie fictive ou authentique des discours ». Il fait ainsi référence aux « linguistes qui, dans la continuité des travaux du post-formaliste russe Mikhaïl Bakhtine, considèrent le dialogue comme une structure sociologique ou translinguistique du langage, mais qui ne s'identifie en aucun cas au dialogue comme genre littéraire ».

Stéphane Pujol retient en outre trois critères pour caractériser la prose d'idées :

«-un discours spéculatif à finalité pédagogique ou philosophique ;

-une rhétorique de type argumentatif (convaincre) plutôt que littéraire (émouvoir) ;

-l'absence de fiction"

Or le titre complet de l'ouvrage que nous étudions est Mémoires d'un père pour servir à l'instruction de ses enfants. Il a été publié pour la première fois en 1804 dans les đuvres posthumes de Marmontel qui est mort en 1799. Dès le titre s'inscrit donc la «finalité pédagogique » dont parle Stéphane Pujol, ce qui est sans doute une bonne raison de lire 
ce texte autobiographique sous l'angle de la prose d'idées. L'incipit de ces Mémoires est encore plus clair :

«C'est pour mes enfants que j'écris l'histoire de ma vie; leur mère l'a voulu. Si quelque autre y jette les yeux, qu'il me pardonne les détails minutieux pour lui, mais que je crois intéressants pour eux. Mes enfants ont besoin de retenir les leçons que le temps, l'occasion, l'exemple, les situations diverses par où j'ai passé m'ont données. Je veux qu'ils apprennent de moi à ne jamais désespérer d'eux-mêmes, mais à s'en défier toujours, à craindre les écueils de la bonne fortune, et à passer avec courage les détroits de l'adversité $»^{2}$.

4 Marmontel a été lié au courant philosophique des Lumières, qu'il semble renier dans le second volume de ses Mémoires, quand il doit reconnaître qu'il est peut-être à l'origine du bouleversement de la Révolution française. Ce double mouvement de promotion et de palinodie à propos des idées nouvelles des philosophes permet aussi de faire entrer ce récit dans un type de discours spéculatif à finalité philosophique, où l'on reconnaît un des critères retenus par Stéphane Pujol pour définir la prose d'idées au XVIII ${ }^{e}$ siècle. À ce titre, il sera également intéressant d'étudier comment se met en place une pensée du récit : les choix narratifs déterminent en effet une idéologie qui reste à définir et qui peut être changeante selon les époques où elle s'ancre. D'autre part, nous pourrons montrer qu'une "rhétorique de type littéraire (émouvoir)» peut avoir une grande valeur argumentative et peut servir à convaincre aussi bien qu'un discours plus froid et plus intellectuel. C'est ce qu'Aristote appelait la "preuve pathétique», et nous aimerions mettre en évidence que le pathétique dans ces Mémoires n'est jamais coupé d'un discours idéologique de persuasion plus souterrain, mais jamais absent pour autant. On trouve enfin un dernier trait générique commun entre les Mémoires et la prose d'idées, telle que la définit Stéphane Pujol : c'est l' « absence de fiction ». Le genre des Mémoires est en effet soumis au " pacte autobiographique » que Philippe Lejeune a étudié pour l'autobiographie, d'une part parce que l'auteur s'y soumet à une obligation de sincérité pour ce qui le concerne, d'autre part parce qu'il renvoie à la grande Histoire et à des faits vérifiables par ailleurs, puisque les Mémoires se situent à l'articulation de la vie privée et de la vie publique, et donc de l'histoire individuelle et de «l'Histoire avec sa grande hache », comme dira plus tard Georges Perec, dans W ou le souvenir d'enfance, ouvrage atypique que l'on s'accorde à placer néanmoins dans le cadre des écritures de soi. Nous voudrions montrer que ces Mémoires de Marmontel, dans lesquels il fait la part belle aux dialogues et aux discours, comme le veut ce type de récit, se présentent comme un dispositif dialogique et polyphonique, en particulier parce que la voix narrative n'est pas une voix unique, ni même univoque, ne serait-ce que parce qu'elle se distingue entre "je narré » et "je narrant», comme Philippe Lejeune l'a si bien montré sur l'exemple canonique et fondateur des Confessions de JeanJacques Rousseau. On pourrait enfin trouver comme point de contact entre la prose d'idées et les Mémoires la difficulté qu'il y a à les définir comme genres, aussi bien caractérisés que les autres, jugés plus importants. C'est ainsi que Yasmina FoehrJanssens et Denis Saint-Jacques écrivent : «Des genres existants sont peu théorisés : ainsi la littérature personnelle (mémoires, journal, autobiographie, etc.) est absente dans les arts poétiques, et mal située dans les poétiques scientifiques $»^{3}$.

5 Avant d'aborder notre démonstration proprement dite, nous allons fixer quelques points de repère sur la vie de Marmontel : il est né en 1723 à Bort-les-Orgues. En 1748, il fait jouer sa première tragédie, Denys le Tyran. En 1758, il se voit confier la direction du Mercure de France. En 1759, il est embastillé dix jours pour des vers satiriques dont il ne 
serait pas l'auteur. En 1763, il entre à l'Académie française. Il publie Bélisaire en 1767 ; le chapitre XV sur la religion fait l'objet d'une controverse. En 1772, il est nommé historiographe de France. En juin 1775, il assiste au sacre de Louis XVI à Reims. En 1777, il se marie avec la sœur de l'abbé Morellet. Il devient un "Père-la-Pudeur", pour reprendre l'expression de Jean-Pierre Guicciardi et Gilles Thierrat dans leur introduction. En 1783, il est élu secrétaire perpétuel de l'Académie. En 1792, il quitte Paris, et vit dans une détresse matérielle dans le Limousin. Il est élu sous le Directoire au Conseil des anciens, mais son élection comme parlementaire en 1797 est invalidée ( « coup d'Etat » du 18 fructidor an V). Il meurt en 1799. Ses Mémoires, publiés en 1804 ont été rédigés entre 1792 et 1796 . Ils sont divisés en 20 livres regroupés en deux parties inégales en volume (p. 33-359; p. 361-478). Les onze premiers livres traitent plus spécifiquement de sa vie privée. La fracture de la Révolution s'inscrit dans la composition même de ses Mémoires. Le douzième livre, qui est le premier de la Deuxième partie, commence par cette phrase, que l'on peut lire comme une prétérition : «Je n'écris pas l'histoire de la Révolution »4.

6 Ce qui est très frappant, c'est que le récit de la vie privée s'efface devant le récit des événements historiques dans la deuxième partie, où le temps du récit et le temps de la narration tendent à se rejoindre. C'est comme si Marmontel n'arrivait pas à articuler les deux. Dans la première partie, l'histoire n'est qu'une toile de fond, sur laquelle se dégagent les grands événements de sa vie privée, et elle est surtout une histoire littéraire, aux figures très intéressantes pour le lecteur moderne. Dans la deuxième partie au contraire, l'Histoire fait de Marmontel un chroniqueur qui semble commenter les événements au jour le jour, sans réussir à y inscrire sa petite histoire, et en reniant tout l'acquis des Lumières qu'il semblait défendre dans la première partie. On voit donc bien, sur une étude de macro-structure, comment les Mémoires peuvent être un genre propice à la prose d'idées et à l'affirmation d'une pensée et de ses impensés.

7 Si l'on reprend la définition de "Mémoires » par le Dictionnaire de l'Académie (1694), on voit le lien originel de cette forme de littérature personnelle avec l'Histoire : « relations de faits, ou d'événements particuliers pour servir à l'histoire». Dans leur article du Dictionnaire du littéraire, Annie Cantin et Alain Viala signalent que «l'emploi du mot au pluriel désigne, dès le XVI ${ }^{e}$ siècle, un genre qui participe à la fois de l'histoire (comme héritier des chroniques et annales) et de l'autobiographie, un récit où une personne consigne des faits qu'elle considère comme dignes d'être notés ou comme nécessaires à un témoignage ou une justification». Après avoir mis en évidence la qualité de « témoins de faits historiques » ou d' « acteurs (aristocrates, généraux, ministres) », ils soulignent la différence entre les Mémoires et l'autobiographie :

«La différence se fait, en pratique, parce que les Mémoires sont censés contenir une part de témoignage historique et ne couvrir qu'une partie de la vie de leur auteur [...] Le genre participe du document historique [...] Mais comme ce genre se fonde sur un regard individuel et une visée d'apologie, il doit aussi assumer la subjectivité qui suscite l'écriture de tels textes [...]. Dès lors une tension s'inscrit entre l'historique et l'esthétique : les Mémoires sont un des genres où le lien entre éthique et esthétique est le plus manifeste ».

D'ailleurs, le chapitre que Georges Gusdorf consacre à " Autobiographie et Mémoires » dans le premier volume des Ecritures du moi s'intitule «le moi et le monde $»^{5}$. C'est en effet aux yeux du monde que l'auteur de Mémoires doit faire bonne figure. Se met donc en place toute une stratégie de conviction et de persuasion de la qualité de son ethos, qui n'est pas une préoccupation étrangère à la prose d'idées. 
9 Dans l'écriture des Mémoires, la question du destinataire est donc primordiale. Marmontel semble la résoudre dès le début, quand il écrit: «C'est pour mes enfants que j'écris l'histoire de ma vie ; leur mère l'a voulu ». Pourtant, ses Mémoires ne sont pas un texte à usage unique, et encore moins à usage interne. Il sait qu'il atteindra un autre public, qui lira par-dessus l'épaule des premiers destinataires. Marmontel a donc soin de se donner toujours le beau rôle, non seulement au regard de ses enfants, mais aussi devant la postérité. Selon le mot des éditeurs, il "écrit des scènes dignes de Greuze ». Le texte s'écrit selon les modèles éprouvés de son siècle, favorables aux bons sentiments, à la sensibilité et se heurtant toujours à la difficulté de les mettre en mots. C'est ainsi qu'il évoque dès le début «l'inexprimable tendresse que [s] a famille avait pour [lui] $»^{6}$. Il s'exclame, comme un personnage de théâtre : «Ah ! quel présent nous fait le ciel, lorsqu'il nous donne de bons parents!» Il est mis au collège de Mauriac. L'organisation temporelle du récit ne suit pas strictement l'ordre des événements, elle acquiert une dimension romanesque grâce à la prolepse pathétique à propos de son père : "Peut-être aussi avait-il quelque pressentiment du malheur que nous eûmes de nous le voir ravir par une mort prématurée ; et, en me faisant de bonne heure prendre un état d'une utilité moins tardive et moins incertaine, pensait-il à laisser en moi un second père à ses enfants $»^{7}$. Les quelques souvenirs de collège de Marmontel sont tout à fait conformes à ce que nous savons du goût du siècle pour le pathétique : les larmes $\mathrm{y}$ sont nombreuses, vertueuses, efficaces : «Le régent, touché de mes larmes et de ma bonne volonté, me reçut et dit à mon père de n'être pas inquiet de moi, qu'il était sûr que je ferais bien ${ }^{8}$. Le jeune Marmontel pleure "de reconnaissance» devant les efforts de son maître pour le mettre au niveau. Les larmes circulent des femmes aux hommes, par l'intermédiaire du papier, comme dans tous les bons romans : «Quand je lui communiquai les lettres où l'amour maternel lui exprimait sa reconnaissance, les larmes lui coulaient des yeux ». Victime de l'injustice du père Bis qui a voulu donner le fouet à un rhétoricien, Marmontel excite par une «harangue " de "grands mouvements d'indignation » chez ses condisciples, en usant d'un style déclamatoire qui prouve qu'il connaît ses classiques : «... mais il aime à punir, il aime à s'abreuver de larmes ; et l'innocent et le coupable, tout lui est égal, pourvu qu'il exerce sa tyrannie... » ${ }^{9}$ Comme le signale la note des éditeurs, l'auteur déforme ici à son avantage cet épisode de sa scolarité ; en fait, il aurait été renvoyé du collège de Mauriac, ce qui expliquerait mieux la décision prise par son père de le mettre en apprentissage. Le pathétique a donc bien ce rapport ambigu à la vérité, comme dans les romans, où il est toujours peu ou prou lié à une stratégie de séduction ou de persuasion qui peut aller jusqu'à déformer les faits. Marmontel reconstruit son passé selon des modèles éprouvés et s'invente une jeunesse digne de lui et du personnage vertueux et courageux dont il veut édifier la statue sous les yeux de ses enfants et de son lecteur. Les figures liminaires des Mémoires, et l'utilisation de tous les procédés du pathétique, permettent la constitution d'une figure digne de foi et d'estime, et participent ainsi à l'idéologie du récit et à une stratégie de la persuasion qui le rapproche de la prose d'idées.

$10 \mathrm{Au} \mathrm{XVIII}$ siècle, la vertu est ostentatoire et la joie d'être une famille unie aussi contagieuse que les larmes du malheur. Ce bonheur d'être ensemble ne serait pas complet s'il ne pouvait se rendre sous la forme d'un tableau émouvant, qui rappelle ceux de Greuze :

« Et quand venait la nouvelle année, c'était dans toute la famille un enchaînement

d'embrassades et un concert de vœux si tendres qu'il eût été, je crois, impossible

d'en être le témoin sans en être ému. Figurez-vous un père de famille au milieu 
d'une foule de femmes et d'enfants, qui, tous levant les yeux et les mains vers le ciel, en appelaient sur lui les bénédictions, et lui, répondant à leurs vœux par des larmes d'amour qui présageaient peut-être le malheur qui nous menaçait: telles étaient les scènes que me présentaient ces vacances $»^{10}$.

11 Conformément aux modèles des récits de vie, l'adolescence est aussi l'âge des premières amours, ici celui pour Mlle $\mathrm{B}^{* * *}$ : « Nos promenades tête à tête, nos entretiens les plus intéressants se passaient à imaginer pour moi dans l'avenir des possibilités de succès, de fortunes, favorables à nos désirs ; mais ces douces illusions, se succédant comme des songes, l'une détruisait l'autre, et, après nous en être réjouis un moment, nous finissions par en pleurer, comme les enfants pleurent lorsqu'un souffle renverse le château qu'ils ont élevés " [guillements manquent ?] . Une tante de sa mère s'en mêle : elle

« accabla cette fille aimable de reproches les plus injustes, sans y épargner les mots d'indécence et de séduction. Après cet imprudent éclat, elle partit et nous laissa, moi furieux et mon amante désolée, étouffant de sanglots et les yeux pleins de larmes. Jugez quelle fut sur mon âme l'impression de sa douleur ».

Nous voyons alors se constituer sous nos yeux un micro-récit qui pourrait fournir le canevas d'une tragédie domestique. Rien n'y manque : gestes expressifs, larmes, grands sentiments, culpabilité et pardon final, dans cette première passion selon Marmontel, où la vertu joue le rôle de deus ex machina, pour mettre tous les personnages d'accord:

«J'avais le front meurtri des coups que je m'étais donnés de la tête contre le mur. Quelle passion que la colère! [...] Ma mère, éperdue elle-même, me serrant dans ses bras et me baignant de larmes, jeta des cris si douloureux que toutes les femmes de la maison, hormis une seule, accoururent ; et celle qui n'osait paraître, et qui venait d'avouer sa faute, s'arrachait les cheveux du malheur qu'elle avait causé ».

Le trop-plein d'émotions doit s'exprimer, s'écouler hors du corps, sous forme de larmes ou de sang:

«Leur désolation, le déluge de pleurs que je voyais pleuvoir autour de moi, ces tendres et timides gémissements que j'entendais m'amollirent le cœur et firent tomber ma colère. Mais j'étouffais, le sang avait enflé toutes mes veines ; il fallut me saigner ».

C'est un dialogue apaisé qui vient clore cette scène pathétique, au terme de laquelle Marmontel donne de lui l'image, pour ne pas dire l'icône, du fils vertueux, ce qui n'est pas anodin dans la constitution d'une idéologie implicite du texte, créée par des moyens esthétiques. Sa mère lui dit: "c'est mon inquiétude sur cette liaison qui a troublé la tête à nos tantes: si tu ne leur pardonnes pas, il faut aussi ne point pardonner à ta mère ». La réponse n'est pas verbale, mais gestuelle : «A ces mots mes bras l'enveloppent et la serrent contre mon cœur ». La tante n'a été méchante que par accident, et même la jeune fille lui a pardonné:

«je viens de l'embrasser: mais elle pleure encore; et vous, qui êtes si bon, ne l'embrasserez-vous pas?»

- Oui, de tout mon cœur ", répondis-je; et dans l'instant la bonne tante vint baigner mon lit de ses larmes. Le soir, le médecin trouva mon pouls encore un peu ému, mais parfaitement bien réglé $»^{11}$.

15 Retour au calme, du cœur, du corps et de la famille après cet accès de pathos qui n'aura eu pour effet que de resserrer des liens vertueux. Qui pourrait croire que Marmontel mènera à Paris une vie bien volage avant de se ranger sur le tard? Dans la scène pathétique, il semble que la vérité compte moins que l'expression d'une vertu triomphante et conforme à des modèles esthétiques auxquels l'auteur cherche à donner 
un fondement éthique. Dans la perspective d'une articulation entre écriture de soi et prose d'idées, il est intéressant de voir que c'est en s'appuyant sur des modèles et des scènes éprouvés dans des genres fictifs (romans, théâtre), que le récit acquiert une dimension éthique qui garantit à la fois sa vérité et la validité de l'idéologie qui émane de lui, et ceci sans rapport exact avec la véracité des faits pris dans leur globalité. Ce n'est pas là un des moindres paradoxes de ces Mémoires, où le je narrant, bon père de famille vertueux, cherche à donner de lui une image bien sage dès la jeunesse, comme pour faire oublier le joli cœur collectionnant les bonnes fortunes qu'il a été. Le pacte autobiographique permet de dissimuler et de faire accepter sans réserves un pacte idéologique qui sans lui demeurerait sujet à caution. Comme la prose d'idées, les Mémoires sont un genre caractérisé par l'absence de fiction, mais c'est paradoxalement par les moyens les plus efficaces de la fiction, injectés dans l'écriture de soi, qu'ils assurent une validité éthique et idéologique au récit. On voit que, comme pour la prose d'idées, le lien à la littérature, s'il est fondamental et déterminant, n'en reste pas moins extrêmement complexe et ambigu.

Le pathétique trouve à s'exprimer partout, en particulier dès que l'écolier prend la plume : «j'écrivis deux lettres [...], l'autre à mon père, où, faisant parler la religion et la nature, je le suppliais avec larmes de ne pas s'opposer à la résolution qui m'était inspirée de me consacrer aux autels $»^{12}$. Il n'hésite pas à convoquer les grands modèles tragiques, sans crainte du ridicule, pour évoquer une scène de collège :

«Je fus devant le père Bis comme Cinna devant Auguste. [...] enfin, mes yeux remplis de larmes osant se lever sur les siens, et voyant qu'il était touché de mon repentir : «Vous me pardonnez donc, mon père? » lui dis-je avec transport; et je me jetai dans ses bras. Je sais bien que les scènes qui nous sont personnelles ont pour nous un intérêt propre qui ne se fait sentir qu'à nous; mais je me trompe ou celle-ci aurait été touchante même pour des indifférents $»^{13}$.

17 Dans ce cas, Marmontel veut nous donner un exemplum de scène pathétique. Ici on peut faire la différence entre un auteur qui, comme Marmontel, ne fait que reproduire les normes et les modes en vigueur, sans prendre aucune distance par rapport à elles, et un véritable créateur comme Rousseau, qui réussit à créer un écart entre le je narrant et le je narré, et à traiter avec humour ou ironie les excès de sa jeunesse et les modèles héroïques qu'il se donnait alors. Il semble que Marmontel rédige ses Mémoires avec les mêmes modèles et les mêmes références que dans ses devoirs d'écolier, comme si l'écrivain n'était jamais qu'un bon élève :

«Le sujet [d'amplification] qu'il me proposa [fut]: les regrets et les adieux d'un écolier qui quitte ses parents pour aller au collège. [...] Je me rappellerais encore l'expression que je donnai aux sentiments du fils et de la mère. Ces mots dictés par la nature, et dont l'art n'imite jamais l'éloquente simplicité, furent arrosés de mes larmes, et le préfet s'en aperçut $»^{14}$.

$18 \mathrm{Au}$ lieu de chercher à mettre en évidence ce qui fait la spécificité de l'enfance et de la jeunesse, il se montre déjà en petit adulte, capable de tirer les leçons de l'amère expérience, conformément au précepte grec selon lequel « la douleur instruit » (pathos mathein), comme en témoigne le dialogue suivant :

«-Hé! mon enfant, me dit-il, à votre âge, le moyen de se faire écouter, obéir, respecter parmi ses pareils ? Vous avez à peine quinze ans

- Il est vrai; mais mon père, ne comptez-vous pour rien le malheur et son influence? croyez-vous qu'il n'avance pas l'autorité de la raison et la maturité de l'âge ? $»^{15}$. 
19 La scène la plus pathétique est celle que provoque la mort du père, que Marmontel avait déjà évoquée dans une prolepse. L'auteur en dramatise l'annonce, en la faisant coïncider avec la fin d'un cycle scolaire :

«Ce fut le jour où je venais de terminer, par cet exercice public [la soutenance des thèses générales], le cours de ma philosophie que j'appris l'événement funeste qui nous plongeait, ma famille et moi, dans un abîme de douleur. [...]

"C'est un malheur bien plus cruel, qui me reste à vous annoncer. Vous n'avez plus de père. " Je tombai sous le coup et je fus un quart d'heure sans couleur et sans voix. Rendu à la vie et aux larmes, je voulus partir sur-le-champ pour aller sauver du désespoir ma pauvre mère $»^{16}$ (p. 62).

C'est le moment d'un véritable morceau de bravoure pathétique pour Marmontel, qui travaille son style pour rendre toute l'horreur de sa situation et toute sa grandeur d'âme. Il faut citer tout le passage, pour montrer comment il convoque des modèles romanesques, dramatiques et picturaux afin de rendre tout le pathétique de cette mort :

« Durant ce funèbre voyage, une seule pensée, un seul tableau présent à mon esprit, l'avait occupé sans relâche et toutes les forces de mon âme s'étaient réunies pour en soutenir l'impression ; mais bientôt, en réalité, il fallut avoir le courage de le voir, de le contempler dans ses plus lugubres horreurs.

J'arrive, au milieu de la nuit, à la porte de ma maison. Je frappe, je me nomme, et, dans le moment, un murmure plaintif, un mélange de voix gémissantes se fait entendre. Toute la famille se lève, on vient m'ouvrir; et, en entrant, je suis environné de cette famille éplorée ; mère, enfants, vieilles femmes, tous presque nus, échevelés, semblables à des spectres, et me tendant les bras avec des cris qui percent et déchirent mon cœur. Je ne sais quelle force, que la nature nous réserve, sans doute, pour le malheur extrême, se déploya tout à coup en moi. Jamais je ne me suis senti si supérieur à moi-même. J'avais à soulever un poids énorme de douleur; je n'y succombai point. J'ouvris mes bras, mon sein, à cette foule de malheureux; je les y reçus tous, et avec l'assurance d'un homme inspiré par le ciel, sans marquer de faiblesse, sans verser une larme, moi qui pleure facilement : " Ma mère, mes frères, mes sœurs, nous éprouvons, leur dis-je, la plus grande des afflictions; ne nous y laissons point abattre. Mes enfants, vous perdez un père ; vous en retrouvez un; je vous en servirai ; je le suis, je veux l'être ; j'en embrasse tous les devoirs; et vous n'êtes plus orphelins ». A ces mots, des ruisseaux de larmes, mais de larmes bien moins amères, coulèrent de leurs yeux. [...] Et mes frères, mes sœurs, mes bonnes tantes, ma grand-mère tombèrent à genoux. Cette scène touchante aurait duré le reste de la nuit, si j'avais pu la soutenir. J'étais accablé de fatigue, je demandais un lit. " Hélas, me dit ma mère, il n'y a dans la maison que le lit de ... » Ses pleurs lui coupèrent la voix. « Eh bien! qu'on me le donne, j'y coucherai sans répugnance. » J'y couchai. Je ne dormis point : mes nerfs étaient trop ébranlés. [...] Ma peau semblait avoir été teinte dans le safran.

Le médecin [...] lui dit que c'était un effet des grandes douleurs concentrées ${ }^{17}$.

21 Tous les procédés se conjuguent ici pour donner à ce passage sa tonalité pathétique : le superlatif, le présent de narration qui met littéralement la scène sous les yeux du lecteur, l'allusion intertextuelle à l'empereur Auguste dans Cinna, les larmes, la phrase de dialogue interrompue par la réticence, la gestuelle qui rappelle les tableaux de Greuze. Ce n'est plus Le Fils puni, mais l'apothéose du fils en père retrouvé. La souffrance s'exprime en signes verbaux ou physiques qui font «tableau " et se manifestent par des phénomènes qu'on n'appelle pas encore psychosomatiques. Le pathétique semble informer toute cette page qui est presque la dernière du livre I et forme ainsi une ponctuation forte du récit. S'il s'est démodé et ridiculisé à nos yeux de 
lecteurs modernes, il était au XVIII ${ }^{\mathrm{e}}$ siècle un gage de succès et une preuve de réussite à la fois littéraire et morale.

Si Marmontel insiste ainsi sur son portrait en orphelin et sur la mort de son père, plus généralement sur la constitution d'une famille vertueuse et unie, ce n'est pas uniquement pour faire concurrence aux grandes fictions du siècle. Ce Livre liminaire de ses Mémoires va permettre de comprendre et de juger, selon un point de vue et une perspective qu'il dessine secrètement, mais non moins fermement dès le début, tous les événements de la Révolution, et en particulier tout ce qui touche la famille royale, dans le second volume de son récit. La prise de position idéologique se fait donc grâce à des choix narratifs apparemment anodins, mais qui appellent une cohérence de lecture telle que le lecteur se trouve pris et entrainé dans une pensée du récit. Après la mort du roi, c'est tous ses ci-devant sujets qui se retrouvent orphelins de père : le régicide est donc assimilé, par les moyens du récit et de sa construction à un véritable parricide. La séparation entre les deux parties des Mémoires est très fortement dramatisée à la fin du Livre XI et de la première partie :

«Bientôt les intérêts de la chose publique et les inquiétudes sur le sort de l'Etat s'emparèrent de mes esprits ; ma vie privée changea de face, et prit une couleur qui nécessairement va se répandre sur le reste de mes Mémoires $»^{18}$.

Les événements publics et historiques semblent réduire au silence le chroniqueur de la vie privée qui devient un témoin de ce qu'il ne peut considérer qu'avec horreur. La deuxième partie sera donc à lire comme une palinodie en creux, où l'auteur de Bélisaire, qui a refusé de plier devant la censure de la Sorbonne, prend la défense du trône et de l'autel, et ne veut pas reconnaître, dans tous les événements sanglants de la Révolution, sinon la conséquence logique, du moins la suite chronologique, des avancées idéologiques obtenues par les encyclopédistes dont il a fait l'éloge pendant toute la première partie :

« Mais si la vie de l'homme est un voyage, puis-je vous raconter la mienne sans dire à travers quels événements et par quels torrents, quels abîmes, quels lieux peuplés de tigres et de serpents elle a passé ? Car c'est ainsi que je me retrace les dix années de nos malheurs, presque en doutant si ce n'est pas un violent et funeste songe. Cette effroyable calamité sera partout décrite en traits de sang: les souvenirs n'en sont que trop ineffaçables $»^{19}$.

On pourrait voir émerger ici un pathos spécifique aux Mémoires sur la Révolution, car on trouverait des images semblables chez d'autres mémorialistes comme l'abbé Morellet ou Mme Campan. Ici le pathétique est mis au service d'une vision péjorative de la Révolution et d'une dénonciation idéologique de ses acteurs et de ses excès. En parlant de «nos malheurs », Marmontel se place résolument du côté de la monarchie, dont il fait une sorte d'extension de sa propre famille, car la formule, s'adressant primitivement à ses enfants, ne manque pas d'ambiguïté. Le mémorialiste fait cause commune avec l'ordre ancien dont il va raconter l'effondrement. A propos de la retraite de Necker le 19 mai 1781, Marmontel fait ce commentaire où il emploie à nouveau un possessif pluriel qui décrit sans doute une famille élargie au-delà de ses enfants pour englober toutes les victimes de la Révolution:

"Voilà de quelle source ont dérivé tous nos malheurs. Nous allons les voir se grossir et se déborder par torrents jusqu'à nous entraîner dans la plus profonde ruine $»^{20}$.

25 A la fin du livre XIV, après avoir dit son aversion pour le régime démocratique, et fait porter la culpabilité de la Révolution sur l'éloquence démagogique du barreau, Marmontel décrit tous les espoirs que la première assemblée portait avec elle : 
«Le roi au milieu de cette cour nationale, plein d'une douce et crédule joie, et autour de lui sa famille heureuse du même bonheur, tout cela, dis-je, ensemble, fit sur les âmes une impression si vive et si profonde que des larmes involontaires coulèrent de tous les yeux. On croyait voir les espérances précéder la marche des Etats généraux, et les prospérités la suivre. Mais au milieu de cet appareil de patriotisme et de concorde, le mouvement sourd qui précède les dissensions orageuses agitait déjà les esprits $»^{21}$.

Il faut se rappeler alors comment Marmontel constitue de lui-même une image de bon père tout au long du livre $\mathrm{X}$, après avoir raconté son mariage avec Mlle de Montigny, dans des pages qui pourraient figurer dans l'Histoire de la vie privée dirigée par Philippe Ariès :

"Je n'oublierai jamais le moment où, dans le jardin de notre petite maison, mon enfant qui n'avait encore osé marcher sans ses lisières, me voyant à trois pas de lui à genoux, lui tendant les mains, se détacha des bras de sa nourrice, et d'un pas chancelant mais résolu vint se jeter entre mes bras. Je sais bien que l'émotion que j'éprouvai dans ce moment est un plaisir que la bonne nature a rendu populaire. Mais malheur à ces cœurs blasés à qui, pour être émus, il faut des impressions artificielles et rares. Une femme de nos amis disait de moi assez plaisamment : « Il croit qu'il n'y a que lui au monde qui soit père $\aleph^{22}$.

27 C'est ainsi que par des effets de composition et de macrostructure, les pages apparemment les plus anodines et les plus particulières, prennent une dimension idéologique et participent de l'élaboration d'une pensée et d'un point de vue, au prix même de quelques reniements. Dans le tableau de Louis XVI en bon père, les larmes acquièrent un statut politique et idéologique. «Involontaires » et unanimes, elles sont la preuve que le monde pourrait continuer à aller comme il va, que chacun y est à sa place, puisque les sujets du roi semblent être une extension de «sa famille heureuse ». Quant au «mouvement sourd » auquel il fait référence, il ne veut pas voir qu'il y a en quelque sorte contribué, en prenant position pour les philosophes, et en faisant sa carrière sous leur tutelle; mais il cherche à faire croire qu'il surgit ex nihilo au milieu de cette foule vertueuse et de bonne volonté. Le pathétique n'est donc pas à traiter uniquement comme un registre ou une tonalité. Envisagé sous l'angle de la prose d'idées et d'une pensée qui s'élabore et cherche à s'imposer au destinataire, dans ce texte adressé que constituent les Mémoires, le pathétique est une sorte de point aveugle qui permet une palinodie salvatrice, proche toutefois de l'aveuglement. Dans cette perspective, il faudrait analyser les stratégies de persuasion dans cette prose d'idées particulière, aussi et peut-être d'abord comme des stratégies d'auto-persuasion.

Le pathétique a aussi une dimension idéologique, comme attachement à l'ordre ancien de la monarchie, quand, au livre XV, Marmontel fait le récit de la journée du 27 juin 1789 qui voit la « réunion des trois ordres dans la salle commune des Etats généraux » :

"Cette réunion solennelle se fit d'abord dans un profond silence. Mais, lorsqu'elle fut consommée, à ce silence respectueux succéda tout à coup une explosion de joie qui se communiqua et se répandit au dehors.

Le peuple, susceptible encore de sentiments honnêtes et de douces émotions, vient d'apprendre que son triomphe est l'ouvrage du roi ; et doublement heureux de l'obtenir et de le lui devoir, se presse vers ce palais, où quelques jours auparavant l'avaient emporté ses alarmes. Il le fait retentir du vœu le plus doux des Français. Il demande à voir ce bon roi, à lui montrer comme il sait l'aimer, à le rendre témoin des transports qu'il lui cause.

Le roi paraît sur le balcon de son appartement, la reine est avec lui ; et tous les deux entendent leurs noms retentir jusqu'au ciel. De douces larmes coulent dans leurs embrassements, et par un mouvement dont tous les cœurs sont attendris, la reine 
serre dans ses bras l'objet de leur reconnaissance. Alors ce peuple qui depuis s'est montré si féroce, et qui était encore bon (j'aime à le répéter), saisit l'instant de payer à la reine ses sentiments d'épouse par un bonheur de mère. Il lui demande à voir son fils, il demande à voir le dauphin. Ce précieux et faible enfant, porté dans les bras de la reine, est présenté par l'amour maternel à la tendresse nationale ; heureux de ne devoir pas vivre assez pour voir quels seraient les retours de cette trompeuse faveur $»^{23}$.

Cette scène très célèbre est racontée au présent de narration, ce qui la met à volonté sous les yeux du lecteur qui en devient un nouveau spectateur, et que Marmontel semble vouloir inclure dans cette foule bienveillante. Une fois encore, les larmes ont une signification idéologique et politique où triomphent les vertus et les bons sentiments de l'Ancien Régime. Le peuple est magnifié, alors que Marmontel saura ailleurs critiquer sa versatilité et son incapacité à se gouverner. Marmontel continue à le percevoir comme une assemblée de sujets et ne veut pas comprendre que, constitué en Tiers état, il est aussi devenu une force politique. La France apparaît comme une grande famille, gouvernée elle-même par une famille vertueuse. Marmontel veut faire croire à la popularité inentamée de Marie-Antoinette, alors qu'elle fait l'objet de très vives critiques dans les pamphlets. Marmontel veut accréditer l'image idyllique d'une osmose bienveillante entre la monarchie et ses sujets, sans prendre en compte l'importance de l'opinion publique, qui est devenue une force politique nouvelle, dont il est difficile de prévoir les réactions, et qu'il a contribué à faire naître. N'est-ce pas en effet en s'appuyant sur elle, qu'il a résisté à la censure de la Sorbonne, lorsqu'elle voulait lui faire modifier le chapitre XV de Bélisaire, comme il le raconte au livre VIII ? Le livre fut imprimé en février 1757. Dans cet épisode encore une fois, Marmontel se donne le beau rôle, en se mettant en valeur dans son attitude philosophique, alors qu'il fit en réalité de nombreuses concessions à la censure :

"Aussitôt qu'il parut, la Sorbonne fut en rumeur, et il fut résolu par les sages docteurs que l'on en ferait la censure. Pour bien des gens, cette censure était encore une chose effrayante, et de ce nombre étaient plusieurs de mes amis. L'alarme se mit parmi eux. Ceux-là me conseillaient d'apaiser, s'il était possible, la furie de ces docteurs; d'autres amis, plus fermes, plus jaloux de mon honneur philosophique, m'exhortaient à ne pas mollir. Je rassurai les uns et les autres, ne dis mon secret à aucun et commençai par bien écouter le public.

Mon livre était enlevé ; la première édition en était épuisée ; je pressai la seconde ; je hâtai la troisième. Il y en avait neuf mille exemplaires de répandus avant que la Sorbonne en eût extrait ce qu'elle devait y censurer, et grâce au bruit qu'elle faisait sur le quinzième chapitre, on ne parlait que de celui-là $»^{24}$.

Il rédige un « résumé » des causes de la censure et le fait « répandre à la ville, à la cour, au parlement, dans les conseils", ce qui rend «la Sorbonne odieuse ». Or la Sorbonne accusait son livre d'être "ennemi du trône et de l'autel »" Marmontel semble avoir oublié ces pages quand il rapporte les scènes publiques d'attendrissement général, où " le peuple " se montre prêt à obéir à ses souverains et à les vénérer indéfiniment. Lui qui connait la force malléable de l'opinion publique, il semble considérer qu'elle s'est évaporée depuis que la liberté n'est plus un combat de philosophes, mais, comme le bonheur selon Saint-Just, « une idée neuve » qui déplace les foules et les fait agir. C'est toute l'ascension sociale de Marmontel qui se trouve ici prise au piège et mise à nu dans ses contradictions et ses compromissions.

31 On choisira la présentation de la figure de Rousseau comme dernier exemple de la manière dont le récit et sa construction dramatisent le débat d'idées tout en le déterminant et en le piégeant. En construisant son image de bon père de famille, 
comme nous l'avons montré plus haut, il dénonce de manière plus souterraine, et dans le creux du texte, Rousseau qui constitue par ailleurs une des cibles principales de ses Mémoires. Marmontel se livre ainsi à un véritable réquisitoire contre Rousseau :

«Le dirai-je? Il faut être époux, il faut devenir père pour juger sainement de ces vices contagieux qui attaquent les mœurs dans leur source, de ces vices doux et perfides qui portent le trouble, la honte, la haine, la désolation, le désespoir dans le sein des familles.

Un célibataire, insensible à ces afflictions qui lui sont étrangères, ne pense ni aux larmes qu'il fera répandre ni aux fureurs et aux vengeances qu'il allumera dans les cœurs [...]

Je blâmais donc Rousseau, mais en le blâmant je m'affligeais que de tristes passions, un sombre orgueil et une vaine gloire eussent gâté le fonds d'un si beau naturel ${ }^{26}$.

Il cherche ensuite à accréditer la thèse selon laquelle le citoyen de Genève se serait suicidé : "après avoir empoisonné ses jours, je ne suis point surpris qu'il en ait volontairement abrégé la triste durée $\aleph^{27}$. Ici le mémorialiste se fait colporteur de ragots qui discréditent son ennemi et, par comparaison, contribuent à conférer à l'auteur une vertu à la mesure des valeurs triomphantes au XVIII ${ }^{e}$ siècle. Même quand il semble gratuit, le pathétique n'est donc jamais idéologiquement neutre; il est toujours orienté en vue d'une stratégie argumentative, ouverte ou secrète, et d'une manipulation du lecteur. L'écriture de soi peut être le lieu d'un débat d'idées qui prend parfois la forme de la polémique en ayant recours à des arguments ad hominem assez contestables, aussi bien du point de vue de la vérité des faits que du point de vue de l'éthique.

Cette dramatisation du débat d'idées par les moyens du récit, de façon aussi bien macrostructurale que microstructurale, entraîne un découpage stratégique des différents livres, avec la recherche d'un effet de suspens. C'est le cas entre le livre VII et le livre VIII. Marmontel dramatise le récit de la rupture entre Rousseau et Diderot, en prenant nettement le parti du second, comme le montrait déjà le récit tendancieux de l'illumination de Vincennes; selon Marmontel, Rousseau aurait choisi le paradoxe par opportunisme ${ }^{28}$. Le livre VII se termine par une question de Marmontel et par le début de la réponse de Diderot:

«Mais dites-moi par quelle rage et sur quel prétexte il vous a si cruellement outragé.

Retirons-nous, me dit-il [Diderot], dans cette allée solitaire : là, je vous confierai ce que je ne dépose que dans le sein de mes amis $»^{29}$.

34 Et le récit de Diderot à proprement parler ne commence qu'au Livre VIII. Créer du suspens dans le débat d'idées est une façon de le truquer en masquant ses partis pris et tous les présupposés qui sous-tendent la polémique. Le récit et ses moyens spécifiques peuvent donc se mettre au service de la mauvaise foi, tout en la dissimulant. Cette attitude n'est pas absente de certains textes plus canoniques de la prose d'idées au XVIII ${ }^{e}$ siècle, en particulier de certains articles de Voltaire, ou de certaines de ses nombreuses lettres.

En somme, poser la question de l'articulation entre l'écriture de soi et la prose d'idées en s'appuyant sur l'exemple des Mémoires de Marmontel, c'est s'interroger plus globalement sur la possibilité d'une pensée des larmes, si l'on peut dire. Il s'agit de savoir si, quand on pleure ou que l'on fait pleurer, on peut encore penser. Dans ce XVIII ${ }^{e}$ siècle qui vit triompher la raison des Lumières, mais aussi le goût des larmes du public, il semble bien que le pathétique se soit mis au service de stratégies argumentatives extrêmement retorses et efficaces. Le récit de Marmontel fournit un 
exemple très particulier de dialogisme, en particulier dans sa construction qui oppose, très schématiquement, la vie privée dans la première partie et la vie publique dans la seconde, en faisant entendre deux jugements contradictoires par la même voix, incapable paradoxalement de voir dans les événements de la Révolution le résultat des combats idéologiques de tout le siècle. Si comme l'affirme Diderot, dans le Paradoxe sur le comédien, "les larmes du comédien coulent de son cerveau ", il semble que les idées du philosophe aient la même source que ses larmes. Tenir le registre de ses émotions et de sa vie privée est donc une pratique qui a à voir avec l'élaboration intellectuelle et spéculative, car c'est une façon d'instaurer un dialogue avec toutes les figures importantes d'un siècle auquel Marmontel ne survivra pas, puisqu'il meurt en 1799.

\section{NOTES}

1. Stéphane Pujol, «La prose d'idées, l'essai et le dialogue philosophique », dans Histoire de la France littéraire, dir. Jean-Charles Darmon, Michel Delon, volume 2,

«Classicismes », PUF, 2006, p. 701-731.

2. Jean-François Marmontel, Mémoires [1804], édition établie, présentée et annotée par Jean-Pierre Guicciardi et Gilles Thierriat, Mercure de France, 1999, p. 35. On ne mettra désormais en note que le numéro de la page.

3. Dictionnaire du littéraire, PUF, 2002.

4. P. 363.

5. Odile Jacob, 1990, p. 239-274.

6. P. 36.

7.P. 39.

8. P. 40.

9. P. 47.

10. P. 50.

11. P. 55.

12. P. 56.

13. P. 57.

14. P. 58.

15. P. 59.

16. P. 62.

17. P. 63.

18. P. 359.

19. P. 363.

20. P. 375.

21. P. 415-416.

22. P. 334.

23. P. 425-426.

24. P. 280.

25. P. 283.

26. P. 336. 
27. P. 338.

28. Voir Livre VII, p. 241-242.

29. P. 268.

\section{RÉSUMÉS}

Poser la question de l'articulation entre l'écriture de soi et la prose d'idées en s'appuyant sur l'exemple des Mémoires de Marmontel, c'est s'interroger plus globalement sur la possibilité d'une pensée des larmes, si l'on peut dire. Il s'agit de savoir si, quand on pleure ou que l'on fait pleurer, on peut encore penser. Dans ce XVIIIe siècle qui vit triompher la raison des Lumières, mais aussi le goût des larmes du public, il semble bien que le pathétique se soit mis au service de stratégies argumentatives extrêmement retorses et efficaces. Le récit de Marmontel fournit un exemple très particulier de dialogisme, en particulier dans sa construction qui oppose, très schématiquement, la vie privée dans la première partie et la vie publique dans la seconde, en faisant entendre deux jugements contradictoires par la même voix, incapable paradoxalement de voir dans les événements de la Révolution le résultat des combats idéologiques de tout le siècle. Si comme l'affirme Diderot, dans le Paradoxe sur le comédien, « les larmes du comédien coulent de son cerveau ", il semble que les idées du philosophe aient la même source que ses larmes. Tenir le registre de ses émotions et de sa vie privée est donc une pratique qui a à voir avec l'élaboration intellectuelle et spéculative, car c'est une façon d'instaurer un dialogue avec toutes les figures importantes d'un siècle auquel Marmontel ne survivra pas, puisqu'il meurt en 1799.

"Autobiographical writing and the prose of ideas, as exemplified by Jean-François Marmontel's Mémoires"

Asking what the relationship is between autobiographical writing and the prose of ideas by using Marmontel's Mémoires as an example amounts to pondering the more general question of the possibility of a so-called teary thought. It is about knowing whether, when one cries or is made to cry, one can still think. During the 18th century, when the public developed a taste for tears despite the triumph of reason in the Age of Enlightenment, it seems that pathos was used for rather crafty and resourceful argumentative purposes. Marmontel's narrative offers a very distinct example of dialogism, particularly in its structure, which very schematically opposes private life in the first part and public life in the second part by making two contradictory opinions heard through the same voice. This voice is paradoxically unable to see the consequences of the century long ideological battles in the events of the French Revolution. If "the actor's tears flow from his intellect", as Diderot claims in the Paradoxe Sur le Comédien, it seems that the philosopher's ideas come from the same source as his tears. As a result, registering one's emotions and private life is intertwined with the intellectual and speculative process, for it is a way to establish dialogue with all the major figures of a century which, because of his death in 1799, Marmontel would not live to see.

\section{INDEX}

Mots-clés : mémoires, ecriture de soi, autobiographie, pathétique, larmes 


\section{AUTEUR}

\section{ANNE COUDREUSE}

Maître de Conférences en Littérature française du XVIIIe siècle à l'Université Paris 13, et membre de l'Institut universitaire de France. Sa thèse est publiée en deux volumes : Le Goût des larmes au XVIIIe siècle (PUF, 1999); Le Refus du pathos au XVIIIe siècle (Champion, 2001). Elle est également l'auteur d'une étude sur Jacques le Fataliste (Gallimard, 1993), des Premières leçons sur La Chute d'Albert Camus (PUF, 1997) et d'un commentaire du Mariage de Figaro (Bordas, 2005). Elle est chroniqueuse dans la revue Les Moments littéraires et travaille actuellement sur les Mémoires de la Révolution. 